\title{
Nematogenic Block Copolymers of Rigid and Flexible Aromatic Units III. Additional Phase Equilibria Studies
}

\author{
E. BIANChi, A. Ciferri, G. Conio, A. TEaldi, \\ W. R. Krigbaum,* and J. Preston* \\ Istituto Chimica Industriale and Centro Macromolecole, \\ C.N.R., University of Genoa, 16132 Genoa, Italy \\ * Department of Chemistry, Duke University, \\ Durham, North Carolina 27706, U.S.A.
}

(Received August 12, 1987)

\begin{abstract}
The mesophase behavior of block copolymers composed of rigid poly $(p$-benzamide) (PBA) and the flexible polyterephthalamide of $p$-aminobenzhydrazide (PABH-T) $(80: 20$, w/w) was investigated using $N, N$-dimethylacetamide (DMAc) $+3 \% \mathrm{LiCl}$ as a solvent. These copolymers include a fraction soluble in dimethyl sulfoxide (DMSO) which was attributed to oligomers and polymers rich in PABH-T. The variation of the volume fraction of the isotropic phase with overall composition within the biphasic gap is more complex than previously observed. As a result, a single anisotropic phase cannot be observed. The flexible component soluble in DMSO is partitioned between the conjugated phases, apparently violating the incompatibility between rigid and flexible polymers in the mesophase. The solubility of these systems is larger than that of the rigid homopolymer. Characterization of the component soluble in DMSO, of the residue, and of the reassembled sample was also performed. The latter shows an incomplete recovery of the properties of the original sample, suggesting labile adducts between rigid and flexible components in the mesophase. Results are discussed in terms of current theories for the liquid crystalline behavior of copolymers.
\end{abstract}

KEY WORDS Block Copolymers / Aromatic Polyamides / Liquid Crystals /

In a previous report ${ }^{1}$ we described the phase behavior of a system obtained by polymerizing $^{2} p$-aminobenzhydrazide and terephthalic acid in the presence of poly(p-benzamide), PBA. The polymerization yielded a mixture of block copolymer composed of rigid PBA and the more flexible polyterephthalamide of $p$ aminobenzhydrazide (PABH-T) sequences, along with some amounts of the flexible and rigid homopolymers. The product resulting from this polymerization exhibited some remarkable properties. It was found that the flexible, PABH-T rich component entered the nematic phase formed above the critical concentration of rigid PBA blocks in $N, N$ dimethylacetamide (DMAc) containing 3\% $\mathrm{LiCl}$. Previous studies ${ }^{3,4}$ concerning mechani- cal blends of PBA and PABH-T homopolymers had demonstrated, in accord with theoretical predictions, ${ }^{5}$ complete exclusion of the flexible homopolymer from the mesophase of the rigid one. Moreover, the solubility of the product of the block polymerization was unusually large. However, when the PABH-T rich component was extracted with dimethyl sulfoxide (DMSO), the solubility of the residue decreased toward the value observed with mechanical blends of the two components.

It was suggested that labile adducts formed during the block polymerization simulated some of the properties expected for a block copolymer. In fact, it has been theoretically predicted $^{6}$ that a block copolymer composed of rigid and flexible blocks, or a mixture of 
such copolymers, can enter the nematic phase. Typical phase diagrams were presented ${ }^{1}$ to illustrate this case.

From a technological standpoint, the increase in solubility with respect to homopolymer PBA, and the occurrence of a flexible component in the mesophase, could have significant relevance. It is well known that the mechanical properties of rigid polymers, such as poly ( $p$-phenylene terephthalate), are considerably improved when the concentration of the nematic dope is increased from 10 to $20 \%{ }^{7,8}$ Moreover, the formation of composites involving rigid and flexible chains is one of the major goals in extending the applications of ultra-high modulus polymers. ${ }^{9}$

This paper presents new phase equilibrium data needed for a better understanding of the behavior of these systems. In particular, we follow the evolution of the composition of the conjugated anisotropic and isotropic phases, ${ }^{3.4}$ using the phase volume ratio, ${ }^{10}$ with the intent of assessing whether a single nematic phase can exist below the solubility limit. We also follow the partitioning of the rigid and flexible components within the conjugated phases in order to analyze the origin of their compatibility in the anisotropic phase. Finally, we explore the reassembly of the two components following their separation.

\section{EXPERIMENTAL}

Three PBA/PABH-T block copolymers (samples A, B, and C) having similar compositions $(80 / 20$ by weight) were used in these studies. Sample A was synthesized following the two-step method described previously, ${ }^{2}$ in which the procedure of Yamazaki et al., ${ }^{11}$ involving triphenyl phosphite (TPP) was used for the preparation of both the PBA prepolymer and the flexible block:

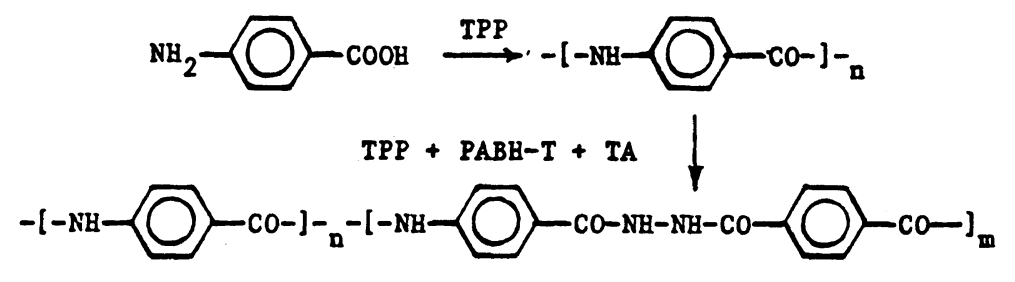

Samples B and C were synthesized following the procedure described by Asrar et al. ${ }^{12}$ using TPP for the formation of the rigid block, and the byproduct diphenyl phosphite (DPP) for the formation of the flexible segment. In other respects, the scheme of eq 1 , was followed. It should be noted that both of these methods have been shown ${ }^{1}$ to produce more homopolymer than the multi-step method.

Solutions were prepared by mixing weighed amounts of dried polymer and DMAc $+3 \%$ $\mathrm{LiCl}$ as the solvent. These mixtures were stirred in sealed tubes at room temperature for at least a week. The polymer concentration, $C_{\mathrm{p}}$, is given as $\mathrm{g}$ polymer per $100 \mathrm{~g}$ of solution. While $C_{\mathrm{p}}$ denotes the overall composition of the bulk sample, $C_{\mathrm{p}}^{\prime}$ and $C_{\mathrm{p}}^{\prime \prime}$ denote the overall com- positions of the isotropic and anisotropic phases, respectively. The latter were determined by precipitating weighed aliquots of each phase. ${ }^{1.3}$ A good separation of the conjugated phases was achieved by centrifuging above $6000 \mathrm{rpm}$ at room temperature for approximately 20 hours. The volume of each phase was determined by calibration of the centrifuge tube. Extraction with DMSO was performed at $80^{\circ} \mathrm{C}$ for about $20 \mathrm{~min}$ for the whole samples, and for the polymer precipitated from the conjugated isotropic and anisotropic phases. ${ }^{3}$ The compositions of the residue and extract were determined by precipitating the polymer with water. Reassembly of the residue and the material precipitated from the extract was performed by dissolution in DMAc $+3 \%$ 
$\mathrm{LiCl}$ in the same proportion established by analysis of the extraction. The overall $C_{\mathrm{p}}$ of $0.9 \%$ should produce an isotropic solution. The reassembled material was recovered by precipitating with water, and washing and drying. The optical anisotropy of the solutions, and the inherent and intrinsic viscosities, $\eta_{\text {inh }}$ and $[\eta]$, were determined as previously described..$^{1-3}$

\section{RESULTS AND DISCUSSION}

\section{Sample Characteristics}

Table I reports the characteristics of the samples. The values of $\left(C_{\mathrm{p}}^{\prime}\right)_{\phi=1}$ refer to the critical concentration of polymer when the volume fraction of the isotropic phase is unity. These values, determined by optical microscopy, are similar for the three samples. They are also rather similar to the value, 7.3, observed for homopolymer PBA having.an inherent viscosity similar to the prepolymer used in making the three block copolymers. On the contrary, considerable variation is shown by the solubility and amount extracted by DMSO. This observation suggests significant differences among the three block polymers. Concerning the solubility data, it should be noted that a considerable role is played by the method of preparation of the saturated solution. For example, by gradually adding small increments of solute, a particular solution reached saturation at $C_{\mathrm{p}}=15.2 \%$. However, when the same polymer and solvent were directly mixed to form a $13.7 \%$ solution, insolubility was observed in spite of prolonged stirring for up to 2 months. A role of the state of agglomeration of the solute was also noticed. These effects are attributed to the exposure of sites for the formation of crystallosolvates, which assist the solubility of PBA in this solvent. ${ }^{8,13}$ The solubility data reported were obtained by the successive addition of small increments of polymer, as previously described. ${ }^{1}$

It was observed that when the freshly synthesized copolymers were dissolved in $\mathrm{DMAc}+3 \% \mathrm{LiCl}$ and reprecipitated with water, the solid recovered was less than the amount originally dissolved. Figures thus obtained are collected in column 10 of Table I. Similar observations were also made in the previous study of the PBA homopolymer. The last entry in column 10 of Table I indicates that the amount of material soluble in water for the PBA homopolymer is comparable to that for the copolymers. Ultraviolet analysis of the water soluble component of PBA suggest-

Table I. Characteristics of samples

\begin{tabular}{|c|c|c|c|c|c|c|c|c|c|}
\hline \multirow{3}{*}{ Sample } & \multicolumn{2}{|c|}{ Rigid/Flexible } & \multicolumn{3}{|c|}{$\frac{\text { Viscosity }}{\mathrm{dl} \mathrm{g}^{-1}}$} & \multirow{3}{*}{$\frac{\left(C_{\mathrm{p}}^{\prime}\right)_{\phi \rightarrow 1}}{\%}$} & \multirow{3}{*}{$\frac{\begin{array}{c}\text { Solub. } \\
C_{\mathrm{p}}^{\prime \prime \prime}\end{array}}{\%}$} & \multirow{3}{*}{$\frac{\begin{array}{c}\text { Ext. } \\
\mathrm{H}_{2} \mathrm{O}\end{array}}{\%}$} & \multirow{3}{*}{$\begin{array}{c}\text { Ext. }^{d} \text { in } \\
\text { DMSO } \\
\%\end{array}$} \\
\hline & \multirow[t]{2}{*}{$w / w$} & \multirow[t]{2}{*}{$\mathrm{mol} / \mathrm{mol}$} & \multirow{2}{*}{$\begin{array}{c}\text { PBA } \\
\eta_{\text {inh }}{ }^{\mathrm{a}}\end{array}$} & \multicolumn{2}{|c|}{ Copolymer } & & & & \\
\hline & & & & $\eta_{\mathrm{inh}}^{\mathrm{b}}$ & {$[\eta]^{\mathrm{b}}$} & & & & \\
\hline A & $81: 19$ & $91: 9$ & 1.6 & 1.88 & - & 7.0 & 15.3 & 9 & 12 \\
\hline B & $80: 20$ & $90: 10$ & 1.4 & 1.84 & 1.96 & 7.1 & $>13.5$ & 4 & 15 \\
\hline $\mathrm{C}$ & $80: 20$ & $90: 10$ & 1.4 & 1.64 & 1.84 & 7.4 & 18.5 & 5 & 15 \\
\hline PBA & $100 \%$ & $100 \%$ & 1.5 & - & 1.80 & 7.3 & 12.1 & 5 & 0 \\
\hline
\end{tabular}

a In $96 \% \mathrm{H}_{2} \mathrm{SO}_{4}$ at $25^{\circ} \mathrm{C}$.

b Solubility in DMAc $+3 \% \mathrm{LiCl}$ at $25^{\circ} \mathrm{C}$.

c Solubility in DMAc $+3 \% \mathrm{LiCl}$.

d Percent extracted referred to whole copolymer following purification with water. 
ed the presence of PBA oligomers (in fact, it has often been observed that repeated purification resulted in a small increase of $\eta_{\text {inh }}$ for PBA). In previous studies, it was possible to use purified PBA obtained by dissolution and reprecipitation. However, in the present study the water soluble component could not be removed until the PABH-T block had been formed. We note that the water soluble component was soluble also in DMSO.

The data in the last column of Table I refer to the amount of polymer extracted by DMSO following the extraction with water. The extract includes homopolymer $\mathrm{PABH}-\mathrm{T}$, and/or a block copolymer rich in PABH-T. ${ }^{2}$ In fact, while PABH-T is soluble in DMSO up to about $18 \%$ at $25^{\circ} \mathrm{C}$, purified $\mathrm{PBA}$ is completely insoluble in DMSO. The residue of the DMSO extraction is attributed to PBA homopolymer and/or block copolymer. These conclusions are supported by NMR data previously presented. ${ }^{2}$ The observation that the total amount of polymer extracted in DMSO is smaller than the amount of flexible monomer confirms that some block copolymer is definitely present.

\section{Phase Volumes and Overall Composition}

The volume fraction of the isotropic phase, $\phi$, for samples A and B is reported in the column 2 of Table II. Columns 3 and 5 give the total polymer reprecipitated from the conjugated isotropic and anisotropic phases, $C_{\mathrm{p}}^{\prime}$ and $C_{\mathrm{p}}^{\prime \prime}$, respectively. The variation with overall composition of the volume fraction of isotropic phase is plotted in Figure 1, along with the variation of the corresponding compositions of the conjugated phases, $C_{\mathrm{p}}^{\prime}$ and $C_{\mathrm{p}}^{\prime \prime}$. While the value $C_{\mathrm{p}}^{\prime}$ at $\phi \rightarrow 1$ ( $c f$. Table I) was determined microscopically by noting the first appearance of anisotropic droplets in isotropic solutions of increasing $C_{\mathrm{p}},{ }^{14}$ values of $C_{\mathrm{p}}^{\prime}$ and $C_{\mathrm{p}}^{\prime \prime}$ at $\phi<1$ were determined analytically from the isolated phases. As expected, $\phi$ decreases when the overall polymer concentration increases. ${ }^{10,15}$ However, at variance with previous results on the same ${ }^{1}$ and other systems, ${ }^{10,16}$ a strong curvature is exhibited by the $C_{\mathrm{p}} v s . \phi$ dependence, which renders problematic the extrapolation of the value $\left(C_{\mathrm{p}}^{\prime \prime}\right)_{\phi \rightarrow 0}$. The calculations of Moscicki and Williams ${ }^{17}$ predict this type of behavior, and could be used for the extrapolation of $\left(C_{\mathrm{p}}^{\prime \prime}\right)_{\phi \rightarrow 0}$, i.e., the value of the overall composition $C_{\mathrm{p}}$ above which the solution is completely anisotropic. These calculations refer to the case of a rigid homopolymer, and the curvature on the $C_{\mathrm{p}} v s . \phi$ plots is attributed to a length distribution of rigid segments. The more pronounced the curvature, the wider will be the biphasic gap. Since our copolymers are prepared from a PBA prepolymer, it is not clear why their rod length distribution should be different from that of pure PBA (for which almost no curvature is observed $\left.^{10}\right)$. Moreover, at variance with the present results, theoretical treatments of copolymers $^{1,6}$ suggest a narrowing of the biphasic gap and a significant increase of $\left(C_{\mathrm{p}}^{\prime}\right)_{\phi \rightarrow 1}$ when short random coil blocks are connected to rigid rods ( $c f$. Figure 6 of the previous paper $\left.^{1}\right)$. The observed widening of the biphasic region could reflect alterations in the state of aggregation of PBA. ${ }^{18}$ A more likely possibility is the occurrence of a fraction of PABH-T blocks, ${ }^{6}$ or homopolymer, ${ }^{5}$ which will never be able to enter the mesophase (Figure $7 \mathrm{~b}$ of our earlier paper ${ }^{1}$ shows that this is the expected behavior ${ }^{6}$ when, in a ternary system, the length of the flexible block is increased).

We note that the horizontal broken lines in Figure 1 (indicating the solubility limit of samples A and B) are clearly below the extrapolated $\left(C_{\mathrm{p}}^{\prime \prime}\right)_{\boldsymbol{\phi} \rightarrow 0}$. Therefore, a pure mesophase (above the biphasic gap) cannot be achieved in the present system due to the early occurrence of crystallization. Earlier we reported ${ }^{1}$ a tentative phase diagram for the present system having a biphasic gap extending between the limits $\left(C_{\mathrm{p}}^{\prime}\right)_{\phi \rightarrow 1}$ and $\left(C_{\mathrm{p}}^{\prime \prime}\right)_{\phi \rightarrow 0}$. These were obtained by linear extrapolation of $C_{\mathrm{p}} v s . \phi$ plots. 
$\left(C_{\mathrm{p}}^{\prime \prime}\right)_{\phi \rightarrow 0}$ was thus reported slightly below the solubility limit, leaving open the possibility of obtaining a single mesophase. The present results rule out such a possibility. When the

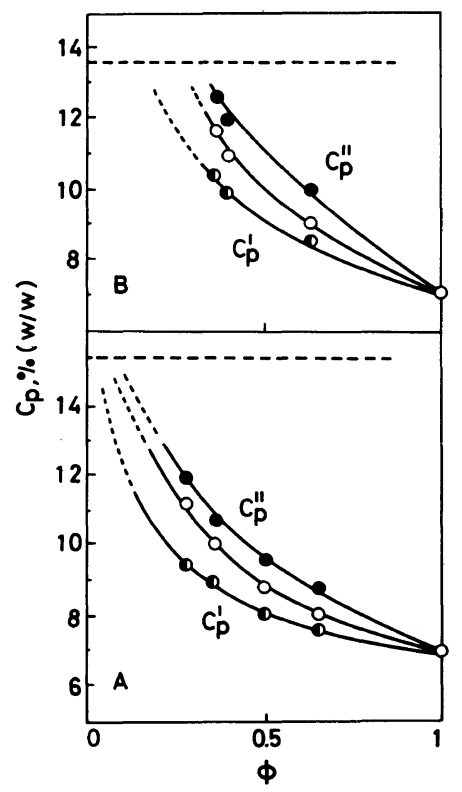

Figure 1. Variation of the composition of the conjugated isotropic, $C_{\mathrm{p}}^{\prime}(\boldsymbol{O})$, and anisotropic, $C_{\mathrm{p}}^{\prime \prime}(\boldsymbol{D})$, phases with the volume fraction, $\phi$, of the isotropic phases for samples A and B. Open circles indicate the overall composition of the biphasic solution. Horizontal dashed lines indicate the solubility limit at room temperature. saturation line crosses the biphasic region, it is possible to observe an equilibrium between isotropic and anisotropic phases $(1>\phi>0)$, but not a single mesophase.

\section{Partitioning}

The conclusion that a single mesophase cannot be attained by the present system weakens our previous suggestion ${ }^{1}$ that the flexible component soluble in DMSO can enter the anisotropic phase. To directly verify this point, one must establish if the DMSO soluble component is effectively partitioned in the anisotropic phase coexisting with the isotropic one.

Table II includes the results of the DMSO extraction performed on the solids precipitated from the conjugated isotropic and anisotropic phases. The weight percent of the DMSOextract reported in the 4 th and 6 th columns refers to the total polymer in each phase. Using these data, we have constructed the diagram in Figure 2. The DMSO extract from each phase, referred to the total extract from the bulk samples, is plotted against $\phi$. Before considering the implication of these results, it is appropriate to verify their consistency with mass balance. Performing the summation:

$$
C_{\mathrm{p}}^{\prime} \phi+C_{\mathrm{p}}^{\prime \prime}(1-\phi)
$$

Table II. Phase volume, composition, and partitioning

\begin{tabular}{|c|c|c|c|c|c|}
\hline \multirow{2}{*}{$C_{\mathrm{p}}($ overall $)$} & \multirow{3}{*}{$\phi$} & \multicolumn{2}{|c|}{ Isotropic } & \multicolumn{2}{|c|}{ Anisotropic } \\
\hline & & $C_{\mathrm{p}}^{\prime \mathrm{a}}$ & DMSO Extract & $C_{\mathrm{p}}^{\prime \prime \mathrm{a}}$ & DMSO Extract \\
\hline$\%(w / w)$ & & $\%(w / w)$ & $E_{\mathrm{x}}^{\prime} \%(\mathrm{w} / \mathrm{w})$ & $\%(w / w)$ & $E_{\mathrm{x}}^{\prime \prime} \%(\mathrm{w} / \mathrm{w})$ \\
\hline \multicolumn{6}{|c|}{ Sample A } \\
\hline 8.04 & 0.65 & 7.6 & 14 & 8.8 & 6 \\
\hline 8.75 & 0.50 & 8.0 & 16 & 9.6 & 7 \\
\hline 10.00 & 0.35 & 8.95 & 17 & 10.7 & 7 \\
\hline 11.20 & 0.28 & 9.4 & 24 & 11.9 & 7 \\
\hline \multicolumn{6}{|c|}{ Sample B } \\
\hline 9.04 & 0.63 & 8.6 & 19 & 10.0 & 10 \\
\hline 10.9 & 0.39 & 9.8 & 25 & 11.9 & 13 \\
\hline 11.66 & 0.36 & 10.4 & 28 & 12.6 & 10 \\
\hline
\end{tabular}

\footnotetext{
a In DMAc $+3 \% \mathrm{LiCl}$.
} 
using the data in the columns 2,3 , and 5 of Table II, the overall $C_{\mathrm{p}}$ in the first column is essentially recovered (justifying the assumption of using $\phi$ instead than a weight fraction). We can also perform the summation:

$$
\left[C_{\mathrm{p}}^{\prime} \phi E_{\mathrm{x}}{ }^{\prime}+C_{\mathrm{p}}^{\prime \prime}(1-\phi) E_{\mathrm{x}}{ }^{\prime \prime}\right] 100 / C_{\mathrm{p}}
$$

where $E_{\mathrm{x}}{ }^{\prime}$ and $E_{\mathrm{x}}{ }^{\prime \prime}$ are the percent DMSO extracts, referred to $C_{\mathrm{p}}^{\prime}$, from the isotropic and anisotropic phases, respectively. The total DMSO extract found in Table $I$ is recovered to within $10 \%$.

It is seen from the data in Table II and Figure 2 that on increasing $C_{\mathrm{p}}$ or decreasing $\phi$, the amount of DMSO-extract partitioned in the anisotropic phase increases, confirming that the more flexible component can enter the anisotropic phase. Interestingly, there seems to be a constant proportion (about $7 \%$ ) of the flexible component to the residue in the latter phase. The simultaneous enrichment of the high molecular weight component in the latter phase is evidenced by the plot shown in Figure 3 of the viscosity of the residue against $\phi$. Fractionation of the rigid component occurring in the residue is in line with previous results for PBA. ${ }^{10}$ However, theoretical predictions $^{6}$ for polydisperse rods connected by flexible segments do not readily explain the observed fractionation. According to Matheson and Flory, ${ }^{6}$ fractionation is inhibited when short random coil blocks are connected to rods. It is possible that our system contains a large portion of PBA homopolymer. Alternative possibilities will be considered below.

Theoretical predictions also offer no basis for the occurrence of flexible homopolymer in the mesophase formed by a rodlike polymer, ${ }^{5}$ or by a rigid rod connected with flexible blocks. ${ }^{6}$ The latter theory ${ }^{6}$ predicts that, for ternary systems, a rigid homopolymer may form a mesophase with a copolymer having rigid and flexible blocks, but in no case will a flexible homopolymer be included. To shed more light on the nature of the DMSO-soluble

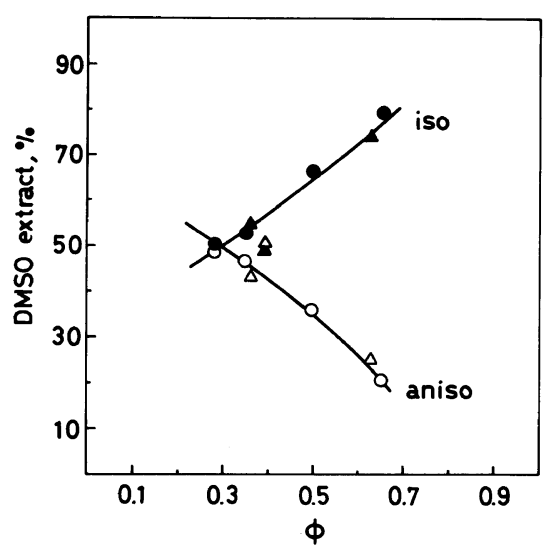

Figure 2. Polymer extracted with DMSO from the conjugated isotropic and anisotropic phases (referred to the total DMSO extract from bulk samples) $v s$. the volume fraction of the isotropic phase. Sample A, O); sample B $(\boldsymbol{\Lambda}, \triangle)$.

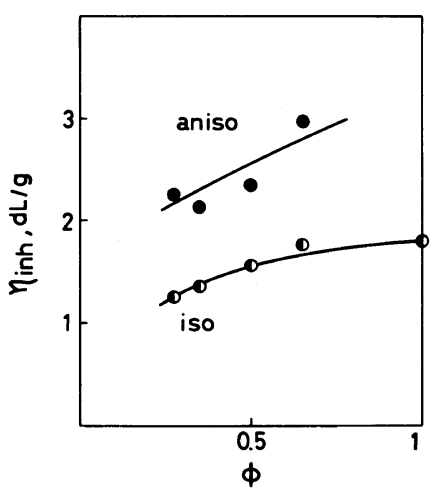

Figure 3. Variation of the inherent viscosity $\left(C_{\mathrm{p}}=\right.$ $0.5 \%$ ) of the residue of the DMSO extract isolated from the anisotropic and isotropic phases of sample A.

component which occurs in the mesophase, we consider the results in the following section.

\section{Reassembly}

In Table III we collect the results for the viscosity, $C_{\mathrm{p}}^{\prime}$ and solubility for whole samples, for the components soluble and insoluble in DMSO, and for the reassembled products resulting from the recombination of extract and residue. The data for sample $\mathrm{A}$ are actually those of the similar sample PX-2 (previously reported $^{1}$ in preliminary form) to 
Table III. Bulk characteristics of isolated and recombined portions of the $80: 20$ block copolymers

\begin{tabular}{|c|c|c|c|}
\hline \multirow{2}{*}{ Sample } & {$[\eta]$} & $\left(C_{\mathrm{p}}^{\prime}\right)_{\phi=1}^{\mathrm{a}}$ & $C_{\mathbf{p}}^{\prime \prime \prime a}$ \\
\hline & $\mathrm{dl} \mathrm{g}^{-1}$ & $\%$ & $\%$ \\
\hline \multicolumn{4}{|c|}{ Sample C } \\
\hline Whole & 1.84 & 7.4 & 18.5 \\
\hline Extract & 0.12 & - & 28 \\
\hline Residue & 1.9 & 6.4 & 14 \\
\hline Reassembled & 1.86 & 7.3 & 15 \\
\hline \multicolumn{4}{|c|}{ Sample $\mathrm{A}^{\mathrm{b}}$} \\
\hline Whole & 1.88 & 7.6 & 15.8 \\
\hline Extract & 0.20 & - & - \\
\hline Residue & - & 7.0 & 12.0 \\
\hline Reassembled & - & - & 10.9 \\
\hline
\end{tabular}

a In DMAc $+3 \% \mathrm{LiCl}$.

b $C_{\mathrm{p}}^{\prime}$ and $C_{\mathrm{p}}^{\prime \prime \prime}$ data for sample $\mathrm{A}$ are those for sample PX-2 in ref 1.

which the determination of $[\eta]$ for sample A has been added. Several aspects of these results deserve consideration. First, the viscosity of the component soluble in DMSO is unexpectedly low. In terms of the $[\eta]-\langle\mathbf{M}\rangle_{v}$ dependence reported for $\mathrm{PABH}-\mathrm{T},{ }^{19}$ molecular weights corresponding to 4 to 7 repeating units are suggested. At the present time we offer no explanation for this finding, although a possible complication might have arisen from the water soluble component resulting from the synthesis of the PBA block, preventing polymerization of long $\mathrm{PABH}-\mathrm{T}$ sequences. Independent tests made to ascertain that PABH-T could be polymerized using the same conditions we had adopted, but without the PBA prepolymer, yielded $[\eta]$ on the order of $3 \mathrm{dlg}^{-1}$. In view of these results, the occurrence of the more flexible component in the mesophase, as shown in Table II, loses some of its postulated significance. ${ }^{1}$ In fact, the expected incompatibility between rigid and flexible polymers is attenuated as the molecular weight of the latter is decreased. ${ }^{5}$ A more quantitative analysis of this effect will, however, be presented elsewhere. ${ }^{20}$
A second interesting feature of the data in Table III is the trend of $C_{\mathrm{p}}^{\prime}$. If the component soluble in DMSO (about $15 \% \mathrm{w} / \mathrm{w}$ ) is regarded as a diluent, the product of $C_{\mathrm{p}}^{\prime}$ of the whole or reassembled samples $(7.3 \%)$ by the weight fraction of the residue yields the correct $C_{\mathrm{p}}^{\prime}$ of the measogens. ${ }^{1}$ We obtain a figure of approximately $6.2 \%$, which is in reasonable agreement with the observed $6.4 \%$ for the residue. The surprising consideration is that the latter figure is significantly smaller than the value, ${ }^{1}$ $7.3 \%$, observed for homopolymer PBA having a similar $\eta_{\text {inh }}$ as our PBA prepolymer (Table I). If the effect is real, it cannot be justified in terms of current theories. ${ }^{5,6}$ In fact $C_{\mathrm{p}}^{\prime}$ of the homopolymer should invariably be increased by the presence of a flexible block ${ }^{6}$ or homopolymer. ${ }^{5}$ A tempting suggestion is the possibility of a conformational reorganization of the flexible block within the mesophase, leading to an increased axial ratio through the selection of more extended conformations. A driving force for such reorganization has only recently been considered. ${ }^{21-24}$

The data in Table III also show interesting effects on the solubility limit. Once the component soluble in DMSO is eliminated, $C_{\mathrm{p}}^{\prime \prime \prime}$ of the whole sample is understandably reduced, but it is surprising that it fails to recover the original value when the sample is reassembled. This observation offers some support to the previous suggestion ${ }^{1}$ that labile adducts between the rigid and the flexible component may occur during the polymerization of PABH-T in the presence of PBA. These adducts would possibly involve the relatively larger molecular weight components of PABH-T, and would not be reformed once the two components are separated and mixed again. Additional support is afforded by the data in Table IV ( $c f$. seq.) However, the limitations (discussed above) on the reproducibility of $C_{\mathrm{p}}^{\prime \prime \prime}$ cast some doubts on these conjectures. We note that the solubility of the extracted component is surprisingly large. ${ }^{3}$ However, we established that it is in line with the 
Table IV. Comparison of partitioning for the whole and the reassembled sample C

\begin{tabular}{|c|c|c|c|c|c|c|c|c|c|}
\hline \multirow{3}{*}{$\frac{C_{\mathrm{p}}{\text { (overall })^{\mathrm{a}}}^{\mathrm{o}}}{\%}$} & \multirow{3}{*}{$\phi$} & \multicolumn{4}{|c|}{ Isotropic } & \multicolumn{4}{|c|}{ Anisotropic } \\
\hline & & \multirow{2}{*}{$\frac{\left(C_{\mathrm{p}}\right)_{\phi=1}^{\mathrm{a}}}{\%}$} & \multirow[b]{2}{*}[\eta]{$^{\mathbf{b}}$} & \multirow{2}{*}{$\frac{\text { Extract }^{\mathrm{c}}}{\%}$} & \multirow[b]{2}{*}[\eta]{$_{\text {Residue }}{ }^{\mathrm{b}}$} & \multirow[b]{2}{*}{$\left(C_{\mathrm{p}}^{\prime}\right)_{\phi=1}^{\mathrm{a}}$} & \multirow[b]{2}{*}[\eta]{$^{\mathrm{b}}$} & \multirow{2}{*}{$\frac{\text { Extract }^{\mathrm{c}}}{\%}$} & \multirow[b]{2}{*}[\eta]{$_{\text {Residue }}$} \\
\hline & & & & & & & & & \\
\hline \multicolumn{10}{|c|}{ Whole sample } \\
\hline 9.39 & 0.58 & 8.6 & 1.30 & 20 & 1.74 & 9.95 & 2.20 & 8 & 2.74 \\
\hline \multicolumn{10}{|c|}{ Reassembled sample } \\
\hline 9.84 & 0.54 & 8.9 & 1.10 & 24 & 1.60 & 10.34 & 2.38 & 4 & 2.68 \\
\hline
\end{tabular}

a In DMAc $+3 \% \mathrm{LiCl}$.

b $[\eta], \mathrm{dl} \mathrm{g}^{-1}$ in DMAc $+3 \% \mathrm{LiCl}$.

c Extract in DMSO referred to the solid precipitated from each phase.

corresponding low value of $[\eta]$ by measuring the variation of $C_{\mathrm{p}}^{\prime \prime \prime}$ with molecular weight for PABH-T.

In Table IV we compare the partitioning of sample $\mathrm{C}$ before and after reassembly. We consider data for a given value of the overall $C_{\mathrm{p}}$ or $\phi$. There is a general similarity between the behavior of the the whole and reassembled samples. However, the amount of the flexible component which enters the anisotropic phase is smaller, by about a factor 2, for the reassembled sample. It is possible that only the component of the DMSO-extract which includes the lowest oligomers (dimers, trimers) can reenter the anisotropic phase due to the reduced incompatibility for low values of molecular weight. ${ }^{5,20} \mathrm{~A}$ remaining portion might have formed labile adducts which are not reformed following separation from the rigid polymer.

\section{CONCLUDING REMARKS}

One of the significant points raised by this series of investigations is the effect of copolymerization on an enhancement of compatibility between mesogens and flexible polymers. The body of the results obtained leaves no doubt that this enhancement does occur. Regretfully, however, the synthetic routes employed failed to give a well defined block copolymer. As a result, we had to struggle with a complex situation based on the coexistence of homopolymers of the rigid mesogen with flexible polymers, oligomers of the latter, and of insufficiently characterized block copolymer. In view of the stimulating results obtained, it is essential that future investigations start with a well characterized block copolymer, to be subsequently investigated as a ternary system involving the rigid or the flexible homopolymer.

The enlargement of the biphasic gap observed with the present systems, which actually prevents the formation of a single mesophase, could be suppressed by avoiding the occurrence of flexible homopolymers unable to enter the mesophase even as labile adducts. Theory predicts a narrow biphasic region and a single mesophase for a binary system involving a block copolymer and a solvent, provided the length of the flexible block is not too large. ${ }^{6}$ The same theory predicts that such a copolymer will form a mesophase at a larger concentration than the corresponding rigid block. However, we observed a smaller critical concentration for the residue of the DMSO extraction than for pure PBA, raising the interesting possibility of a conformational reorganization of the flexible block. Such an effect, if it can be confirmed, would enhance compatibility and would require a new theoretical description of 
these systems.

The partitioning of oligomers of the flexible polymer in the mesophase would not be an advantage from the point of view of enhanced mechanical properties of fibers spun from such a mesophase. However, the occurrence of labile adducts involving larger molecular weight components of the flexible polymer should be of interest. The actual formation of such adducts does require a more convincing demonstration.

The solubility of the present systems is certainly larger than that of the corresponding PBA. Inspection of the data in Tables I and III suggests that the largest contribution to the solubility increase is due to the component extractable with DMSO. When the latter fraction is small (Residue, Table III, $c f$. also data ${ }^{1}$ for the PBA/Nomex copolymer) only a modest solubility increase of the copolymer over the rigid homopolymer is observed.

Acknowledgments. We express our appreciation to Dr. E. Marsano for valuable assistance. This investigation was supported by the Italian Research Council and by the Office of Naval Research, Grant N00014-84-K-0496.

\section{REFERENCES}

1. W. R. Krigbaum, Z. Shufan, J. Preston, and G. Conio, J. Polym. Sci., Polym. Phys. Ed., 25, 1043 (1987).

2. W. R. Krigbaum, J. Preston, A. Ciferri, and Z. Shufan, J. Polym. Sci., Polym. Chem. Ed., 25, 657 (1987).
3. E. Bianchi, A. Ciferri, and A. Tealdi, Macromolecules, 15, 1268 (1982).

4. E. Bianchi, A. Ciferri, G. Conio, E. Marsano, and A. Tealdi, Macromolecules, 17, 1526 (1984).

5. P. J. Flory, Macromolecules, 11, 1138 (1978).

6. R. R. Matheson and P. J. Flory, Macromolecules, 14, 954 (1981).

7. H. Blades, U. S. Patent $3,869,429$ (1975) to Du Pont Co.

8. G. Conio, R. Bruzzone, A. Ciferri, E. Bianchi, and A. Tealdi, Polym. J., 19, 757 (1987).

9. D. Prevorsek, "Polymer Liquid Crystals," A. Ciferri, W. R. Krigbaum, and R. B. Meyer, Ed., Academic Press, New York, N.Y., 1982.

10. G. Conio, E. Bianchi, A. Ciferri, and A. Tealdi, Macromolecules, 14, 1084 (1981).

11. N. Yamazaki, M. Matsuo, and F. Higashi, J. Polym. Sci., Polym. Chem. Ed., 13, 1373 (1975).

12. J. Asrar, J. Preston, A. Ciferri, and W. R. Krigbaum, J. Polym. Sci., Polym. Phys. Ed., 20, 373 (1982).

13. S. P. Papkov, Adv. Polym. Sci., 59, 75 (1984).

14. C. Balbi, E. Bianchi, A. Ciferri, A. Tealdi, and W. R. Krigbaum, J. Polym. Sci., Polym. Phys. Ed., 18, 2037 (1980).

15. P. J. Flory and R. S. Frost, Macromolecules, 11, 1126 (1978).

16. G. Conio, E. Bianchi, A. Ciferri, and W. R. Krigbaum, Macromolecules, 17, 856 (1984).

17. J. K. Moscicki and G. Williams, Polymer, 23, 558 (1982).

18. B. Millaud and C. Strazielle, Makromol. Chem., 179, 1261 (1978).

19. J. J. Burke, J. Makromol. Sci. Chem., A7, 187 (1973).

20. E. Marsano and A. Ciferri, in preparation.

21. P. J. Flory and R. R. Matheson, J. Phys. Chem., 88, 6606 (1984).

22. S. V. Vasilenko, A. R. Kokholov, and V. P. Shibaev, Macromolecules, 17, 2270 (1984).

23. R. E. Boehm, D. E. Martire, and N. V. Madhusvdana, Macromolecules, 19, 2329 (1986).

24. D. Y. Yoon, Intl. Symp. in Approaches to Property Limits in Polymers, A.C.S., Princeton, N.J., Aug., 1986. 